\title{
COMPORTAMENTO DE RISCO PARA A SAÚDE DE ESCOLARES DO ENSINO MÉDIO DE BARRA DOS COQUEIROS, SERGIPE, BRASIL
}

\author{
MS. JADSON DE OLIVEIRA LIMA \\ Mestrado em Saúde e Ambiente pela UNIT e Professor da \\ Universidade Tiradentes, Aracajú, Sergipe, Brasil \\ e-mail: Jadsonjl@hotmail.com \\ DRA. VÂNIA FONSECA \\ Doutora em Geografia/Planejamento Regional pela UNESP, \\ Campus de Rio Claro e Professora do Instituto de Tecnologia e \\ Pesquisa da Universidade Tiradentes, Aracajú, Sergipe, Brasil \\ e-mail:vania@infonet.com.br
}

\section{DR. DARTAGNAN PINTO GUEDES}

Doutor em Educação Física pela EEFE/USP e Professor Associado do Centro de Educação Física e Esporte da Universidade Estadual de Londrina, Paraná, Brasil e-mail: darta@sercomtel.com.br

\begin{abstract}
RESUMO
O objetivo do estudo foi analisar a prevalência de comportamentos de risco para a saúde em escolares do ensino médio das redes pública e privada do município de Barra dos Coqueiros, Illha de Santa Luzia, que recentemente foi ligada a Aracaju, capital do Estado de Sergipe, Brasil. As informações foram coletadas mediante questionário auto-administrado. Dos escolares analisados, 89,2\% referiram ser sedentários; 32,6\% já haviam experimentado cigarro; 41,9\% consomem freqüentemente bebida alcoólica; e 9, 1 \% já usaram algum tipo de droga ilíita. Estes resultados reforçam a necessidade de novos estudos de base populacional, de forma a permitir melhor compreensão das relações entre os comportamentos de risco e 0 ambiente social dos jovens.
\end{abstract}

PALAVRAS-CHAVE: Estilo de vida; adolescência; educação para a saúde. 
A infância e a adolescência representam períodos críticos nos quais vários hábitos de vida são estabelecidos, de modo que muitos dos comportamentos assumidos durante estes períodos da vida tendem a serem mantidos na vida adulta, tornando-se, portanto, de difícil modificação (BRANEN; FLETCHER, 1999). As influências do cotidiano, aliada à transição física e psicológica crítica, típica do período da adolescência, contribuem para a adoção de atitudes associadas ao início precoce e inadequado da prática sexual, ao sedentarismo, ao uso de tabaco, ao consumo de bebida alcoólica e de outras drogas psicoativas. Essas atitudes se configuram na expressão denominada de comportamento de risco para a saúde, definida como comportamento decorrente de opções, atitudes e oportunidades que tendem a exercer impacto negativo no estado de saúde dos jovens, elevando prematuramente o risco de morbimortalidade (OMS, 1999).

No Brasil, são poucos os estudos que procuraram investigar os comportamentos de risco para a saúde em amostras representativas de jovens. Os poucos estudos já realizados procuraram abordar itens isolados de comportamento de risco para a saúde, como consumo de bebida alcoólica (GALDUROZ et al., 2005), uso de tabaco (Malcon et al., 2003; CEBRID, 2006), consumo de drogas ilícitas (CEBRID, 2006), hábitos alimentares inadequados (CASTRO et al. , 2008; CARMO et al., 2006) e sedentarismo (GUEDES et al., 200I).

Com relação ao consumo de bebida alcoólica, estudo em escolares no conjunto das 27 capitais brasileiras apontou que a idade média para a iniciação do álcool é de 12,5 anos (GALDUROZ et al., 2005). Este dado preocupa em razão do consumo precoce de bebida alcoólica se caracterizar com um fator de exposição para problemas de saúde em idade adulta, além de aumentar acentuadamente o risco de se tornar consumidor em excesso ao longo da vida (STRAUCH et al., 2009).

Por outro lado, jovens fumantes possuem elevada probabilidade de se tornarem adultos fumantes, aumentando assim o risco de mobimortalidade por doenças crônicas e causas evitáveis (MALCON et al., 2003). A iniciação precoce ao fumo é um importante preditor de consumo de outras substâncias, como álcool e drogas ilícitas (PEDEN, 2008). Segundo o Levantamento Domiciliar sobre o Uso de Drogas Psicotrópicas no Brasil, realizado em 2005, na faixa etária de 12 a 17 anos, I5,2\% dos jovens faziam uso de tabaco, sendo que 2,9\% usavam habitualmente. A idade média de iniciação ao tabaco foi de 12,8\% (CEBRID, 2006). Ainda, este mesmo estudo aponta que $8,7 \%$ dos jovens entrevistados já haviam usado algum tipo de droga ilícita, como é o caso de maconha, cocaína, crack, cola ou ecstasy.

No que se refere aos hábitos alimentares, estudo realizado na rede pública de ensino da cidade do Rio de Janeiro apontou elevado consumo de alimentos 
não-saudáveis, como doces, refrigerantes, frituras e salgados, e baixo consumo de frutas e hortaliças (CASTRO et al., 2008). Em outro estudo envolvendo escolares de uma cidade do interior do estado de São Paulo revelou que 83,8\% apresentavam ingestão calórica e 36,7\% ingestão de lipídeos acima das recomendações para idade (CARMO et al., 2006). Quanto à atividade física, informações disponibilizadas na literatura mostram que, em média, o tempo semanal dedicado à prática de esporte e exercício físico de escolares do ensino médio é por volta de 50 minutos entre moças e 200 minutos entre rapazes (GUEDES et al, 200 I).

O estudo do conjunto de indicadores relacionados ao comportamento de risco para a saúde da população jovem deve ser fator prioritário quando está em foco a formação do futuro adulto. A análise dos indicadores inter-relacionados aos comportamentos de risco para a saúde poderá subsidiar a implantação de programas de educação e promoção da saúde, no âmbito escolar e populacional, bem como servir de subsídio no monitoramento das características dos comportamentos de risco associados às doenças crônico-degenerativas e de referência para o acompanhamento e a avaliação de eventuais ações intervencionistas que venham a ser desenvolvidas.

Nesse sentido, o presente estudo teve por objetivo analisar a prevalência de comportamentos de risco para a saúde em escolares do ensino médio das redes de ensino público e privado da cidade de Barra dos Coqueiros. Esta cidade é apontada como referência no Estado de Sergipe devido às suas características peculiares. Sua base territorial é uma ilha que sofreu quebra recente do isolamento rodoviário em razão da construção de uma ponte ligando a sede municipal ao centro da capital sergipana. A cidade, ainda em processo inicial de transição, permite não apenas que seja estudada a situação existente durante o seu relativo isolamento geográfico, mas também, o acompanhamento das alterações decorrentes de sua inserção funcional na zona metropolitana de Aracaju.

\section{METODOLOGIA}

estudo, do tipo epidemiológico com delineamento transversal, teve como população de referência os escolares do ensino médio de ambos os gêneros com idades entre 14 e 18 anos, regularmente matriculados nas unidades de ensino médio das redes de ensino público e privado da cidade de Barra dos Coqueiros, Sergipe, Brasil. Os protocolos de intervenção utilizados foram aprovados pelo Comitê de Ética em Pesquisa da Universidade Tiradentes (CEP-UNIT) e acompanharam normas da Resolução 196/96 do Conselho Nacional de Saúde sobre pesquisa envolvendo seres humanos.

O município de Barra dos Coqueiros, com território na llha de Santa Luzia, localizada em frente à Aracaju, embora faça parte da sua região metropolitana, até 
recentemente se ligava à capital do Estado de Sergipe por transporte fluvial. Em 2006-2007 entrou em funcionamento a ponte sobre o rio Sergipe e Barra dos Coqueiros passou a se integrar funcionalmente à metrópole, o que causou grandes modificações para as zonas urbana e rural deste município. Com área reduzida aos limites da ilha, a cidade de Barra dos Coqueiros ocupa 87,9 km², e apresentava densidade demográfica próxima de 220 hab/km² em 2007 (IBGE, 2008). Caracterizado pelo baixo dinamismo econômico até 2005, o município está tendo essa situação bastante alterada, fruto da construção da ligação rodoviária direta à Aracaju. O processo de desenvolvimento pelo qual vem passando, com a apropriação de novos espaços, inclusive na zona rural, para atender o surto imobiliário que se instalou no município, está mudando a configuração não apenas da paisagem, mas das relações sociais que ali têm lugar. E, como consequência dessas modificações, se destaca a questão de segurança que, junto com o novo dinamismo econômico e a chegada de novos empreendimentos e habitantes, é um dos maiores responsáveis pela modificação do cotidiano dos antigos habitantes.

O Índice de Desenvolvimento Humano (IDH), calculado pelo Programa das Nações Unidas Para o Desenvolvimento, informa valor de 0,676 para o ano de 2000, o que coloca o município entre aqueles de médio desenvolvimento humano, especialmente ao considerar que o estado de Sergipe apresentou IDH da ordem de 0,622 e a região Nordeste índice discretamente mais baixo, 0,61 I. Porém, o índice observado em Barra dos Coqueiros ainda está abaixo da média brasileira, que alcançou 0,699 (PNUD, 2003). Para a composição do índice foram significativas a taxa bruta de frequência escolar (87, I 5\%) e a taxa de alfabetização de adultos (79, I 8\%), o que levou o índice de educação (IDHM-E) para 0,8।8, uma vez que, as contribuições da longevidade (IDHM-L =0,63I) e da renda (IDHM-R =0,578) foram baixas.

\section{AMOSTRA E SELEÇÃO DOS SUJEITOS}

A cidade de Barra dos Coqueiros tem três unidades de ensino médio, sendo uma da rede estadual, com 562 escolares matriculados no ano de 2007, e outras duas unidades da rede privada de ensino, uma com 23 e outra com 50 alunos matriculados, o que totaliza uma população de 635 escolares de ensino médio. Considerando intervalo de confiança de $95 \%$, erro amostral de $5 \%$ e critérios de inclusão adotados, estimou-se inicialmente uma amostra de 280 escolares atendendo às características estabelecidas. Vale ressaltar que a quantidade de escolares das unidades de ensino da rede privada foi bastante reduzida e para garantir a representatividade destas unidades de ensino, todos os escolares matriculados e frequentando as aulas foram inseridos na amostra. 
A seleção da amostra ocorreu por processo probabilístico aleatório, com estratificação por turma. $\bigcirc$ sorteio dos alunos ocorreu mediante utilização da relação nominal de todos os matriculados, fornecida pelas unidades de ensino. Para não sacrificar a representatividade amostral foi acrescentado 20\% ao total de sujeitos da amostra, de forma a atender eventuais perda no acesso às unidades selecionadas. Assim, a amostra definitiva envolvida no tratamento das informações foi composta por 353 escolares (203 moças e I 50 rapazes).

\section{COLETA DOS DADOS}

O instrumento de coleta das informações adotado para o estudo foi elaborado a partir da composição de sessões de outros instrumentos já validados e empregados em estudos anteriores envolvendo escolares, contemplando as variáveis: idade, gênero, prática habitual de atividade física, hábitos alimentares, uso de tabaco, consumo de bebida alcoólica e de outras drogas, medidas antropométricas (massa corporal e estatura). O questionário, pré-testado, foi preenchido pelo informante, sem identificação pessoal. Durante a distribuição dos questionários foi esclarecido que os escolares poderiam optar em devolvê-los não-preenchido e, para aqueles que aceitassem responder, garantida a não identificação do informante.

Para o levantamento da prática habitual de atividade física foi utilizado o Physical Activity Questionnaire for Older Children (PAQ-C), sugerido por Crocker et al. ( 1997), traduzido e adaptado para a realidade brasileira por Silva e Malina (2000). A escolha deste instrumento foi motivada tanto pela sua qualidade quanto pela facilidade de ser entendido pelos adolescentes. Este instrumento apresenta valores de consistência interna entre 0,79 e 0,89 e fidedignidade teste-reteste entre 0,75 e 0,82 (CROCKER et al., 1997). O preenchimento do questionário pelos escolares teve duração média em torno de 20 minutos.

O questionário é composto por nove questões relacionadas à prática de atividade física na escola e no lazer em finais de semana, incluindo também, questões quanto ao tempo despendido diante de TV e computador. Cada questão apresenta escores entre I e 5. A classificação da prática habitual de atividade física é baseada no cálculo médio dos escores equivalentes às atividades diárias e semanais. Neste caso, podem-se classificar como ativos aqueles escolares que apresentam escore $\geq 3$ e sedentários os escolares com escore $<3$. $\bigcirc$ índice Kappa do estudo piloto foi considerado adequado para a pesquisa, alcançando valor 0,9।4 em relação ao nível de prática habitual de atividade física estimado pelo PAQ-C.

Para levantamento das informações associadas aos hábitos alimentares utilizouse o questionário apresentado por Farias Júnior (2002), com base em informações 
referentes à frequência de consumo semanal e diário de 6 grupos de alimentos: grupo 1: refrigerantes; grupo 2: leite e derivados; grupo 3: doces; grupo 4: batata frita, salgadinhos, hambúrguer; grupo 5: frutas; e grupo 6: verduras. Para análise dos dados assumiu-se comportamento alimentar saudável o consumo de laticínios, frutas e verduras $\geq 4$ vezes/semana; pouco ou nenhum consumo de refrigerantes, doces, frituras, hambúrguer, cachorro quente e salsicha. Comportamento alimentar pouco saudável, mas aceitável, o consumo de refrigerantes, doces, frituras e hambúrguer/ cachorro quente/salsicha $\leq 3$ vezes/semana. Comportamento alimentar de risco para a saúde o não consumo de laticínios, frutas e verduras, e o consumo $\geq 4$ vezes/ semana de refrigerantes, doces, frituras, hambúrguer, cachorro quente e salsicha.

O levantamento quanto ao uso de tabaco, ao consumo de bebidas alcoólicas e de outras drogas foi realizado por meio de questões disponibilizadas na versão brasileira do Youth Risk Behavior Survey, traduzida e adaptada transculturalmente por Guedes e Lopes (20 I0).

\section{TRATAMENTO ESTATÍSTICO}

Para análise dos dados utilizou-se dos recursos da estatística descritiva envolvendo o cálculo da proporção de ocorrência de cada comportamento de risco considerado, separadamente por gênero. Para verificação de possíveis diferenças entre gêneros foi empregado teste de significância para comparações de proporções mediante envolvimento da estatística de qui-quadrado $\left(\chi^{2}\right)$. Eventuais associações entre os comportamentos de risco para a saúde foram analisadas mediante o cálculo de coeficiente de correlação não-paramétrica de Spearman, assumindo nível de significância estatística de $p<0,05$. O tratamento estatístico foi realizado utilizando o pacote computadorizado Statistical Package for the Social Sciences (SPSS), versão 17.0.

\section{RESULTADOS}

As prevalências encontradas equivalentes aos comportamentos de risco são apresentadas na tabela I. Os resultados apontam elevada proporção de sedentarismo em ambos os gêneros, sendo as moças significativamente mais sedentárias que os rapazes $(p<0,00 \mathrm{I})$. Quanto aos hábitos alimentares, os rapazes relataram proporção significativamente maior quanto ao consumo $\leq 3$ vezes/semana de frutas $(p=0,035)$ e verduras $(p=0,0 \mid 3)$, e ao consumo $\geq 4$ vezes/semana de doces $(p=0,017)$, o que sugere uma alimentação menos saudável que as moças. 
Tabela I. Prevalência (95\% IC) de comportamentos de risco para a saúde de escolares da cidade de Barra dos Coqueiros, Ilha de Santa Luzia, Sergipe, Brasil. 2008.

\begin{tabular}{|c|c|c|c|}
\hline Comportamentos de Risco & Moças & Rapazes & Teste $\chi^{2}$ \\
\hline Sedentarismo & $96,1(94,0-98,3)$ & $75,8(73,9-77,8)$ & $<0,001$ \\
\hline \multicolumn{4}{|l|}{ Hábitos Alimentares } \\
\hline Leite/queijos $\leq 3$ vezes/semana & $73,4(7 ।, 7-75,2)$ & $77,5(75,7-79,4)$ & ns \\
\hline Frutas $\leq 3$ vezes/semana & $86,7(84,8-88,7)$ & $94,2(92,0-96,6)$ & 0,035 \\
\hline Verduras $\leq 3$ vezes/semana & $78,5(76,7-80,4)$ & $89,2(87,|-9|, 6)$ & 0,013 \\
\hline Refrigerantes $\geq 4$ vezes/semana & $54, I(52,6-55,8)$ & $63,3(61,6-65,2)$ & \\
\hline Doces $\geq 4$ vezes/semana & $46,4(45,1-47,9)$ & $60,0(57,9-62,4)$ & 0,017 \\
\hline Frituras $\geq 4$ vezes/semana & $48,0(46,6-49,6)$ & $47,5(45,9-49,4)$ & ns \\
\hline \multicolumn{4}{|l|}{ Uso de tabaco } \\
\hline Experimentação & $32,6(29,5-36,1)$ & $32,5(29,6-35,7)$ & ns \\
\hline Uso frequente & $11,8(10,0-13,9)$ & $0,0(0,0-0,0)$ & $<0,001$ \\
\hline \multicolumn{4}{|l|}{ Consumo de bebida alcoólica } \\
\hline Experimentação & $81,5(77,3-86,0)$ & $83,3(79,4-87,4)$ & ns \\
\hline Consumo frequente & $40,8(37,6-44,2)$ & $43,3(40,4-46,4)$ & ns \\
\hline Experimentação de drogas ilícitas & $8,2(6,3-10,1)$ & $10,8(8,8-12,9)$ & ns \\
\hline Relacionamento sexual & $55,8(52,6-59,4)$ & $75,0(70,9-79,4)$ & $<0,001$ \\
\hline Uso de preservativo & $37,8(33,9-41,9)$ & $55,0(49,9-60,1)$ & 0,003 \\
\hline
\end{tabular}

Esses comportamentos foram acompanhados por outros, também considerados negativos, como uso de tabaco e consumo de bebidas alcoólicas. Os dados mostram que mais de $32 \%$ das moças e dos rapazes já experimentaram cigarros e proporção superior a $80 \%$ dos escolares já experimentaram bebidas alcoólicas. Com relação à experimentação de drogas ilícitas, os dados encontrados apontam que por volta de um em cada dez escolares já experimentou algum tipo de droga que não seja cigarro ou bebidas alcoólicas, enquanto 3, I \% dos escolares optaram por não responder a esta questão. Ainda, verifica-se que a maioria dos escolares informou já ter se relacionado sexualmente, além do que, não mais de $38 \%$ das moças e $55 \%$ dos rapazes usam preservativos nas relações sexuais.

Analisando os valores calculados para o índice de correlação de Spearman (Tabela 2) destacam-se algumas associações estatisticamente significativas. O maior escore de correlação encontrado refere-se à associação entre não uso de preservativos e ocorrência de relacionamento sexual precoce, ou seja, em idades $<13$ anos $(0,505)$, seguido do escore de correlação entre experimentação de tabaco e de outras drogas $(0,307)$. Demais associações apontadas como significativas referemse ao consumo regular de bebidas alcoólicas e não uso de preservativos $(0,259)$; experimentação de drogas $(0,254)$ e relacionamento sexual precoce $(0,21$ I). 
Tabela 2 - Escores significativos $(p<0,05)$ dos coeficientes de correlação de Spearman entre indicadores de comportamento de risco para a saúde em escolares - Barra dos Coqueiros, Sergipe, Brasil. 2008.

\begin{tabular}{lll}
\hline & Variáveis & $\begin{array}{l}\text { Correlação de } \\
\text { Spearman }\end{array}$ \\
\hline Não usa preservativo & Relacionamento sexual precoce & 0,505 \\
Experimentou fumar & Experimentou drogas & 0,307 \\
Consumo de bebida alcoólica & Não usa preservativo & 0,259 \\
Consumo de bebida alcoólica & Experimentou drogas & 0,254 \\
Consumo de bebida alcoólica & Relacionamento sexual precoce & 0,211 \\
\hline
\end{tabular}

\section{DISCUSSÃO}

O estudo procurou identificar a prevalência de selecionados comportamentos de risco para a saúde e suas inter-relações em escolares do ensino médio das redes de ensino público e privado da cidade de Barra dos Coqueiros, Sergipe, Brasil. A elevada proporção de sedentarismo encontrada pode representar maior probabilidade dos escolares permanecerem sedentários na vida adulta e, portanto, não usufruirem do impacto favorável que a atividade física regular possa exercer para um melhor estado de saúde (GUEDES et al., 200 I). Resultados semelhantes foram encontrados em outros estudos que utilizaram metodologia similar (instrumento de medida e estratégias para classificar os adolescentes em função o nível de atividade física), a exemplo do estudo realizado por Silva e Malina (2000) em Niterói, Estado do Rio de Janeiro, que identificou entre jovens de 14 e 15 anos, de ambos os gêneros, prevalência de 85\% dos rapazes e $94 \%$ das moças classificadas como sedentários.

Estudo realizado por Rosa et al. (2007) na cidade de Aracaju, Sergipe, envolvendo escolares de ambos os gêneros com idades entre 12 e 16 anos, encontraram prevalência de sedentarismo de $85,2 \%$ nas moças e 70,8\% nos rapazes. Os resultados relacionados ao sedentarismo foram superiores aos encontrados por Guedes et al. (200 I), em estudo realizado em Londrina, Paraná, envolvendo escolares de ambos os gêneros, com idades entre 15 e 18 anos, que identificou $65 \%$ das moças como inativas e muito inativas fisicamente e $54 \%$ dos rapazes como ativos e moderadamente ativos.

Em outro estudo realizado por Santos e Silva (2007), também no município da Barra dos Coqueiros, Sergipe, com a participação de escolares de I I- I3 anos de ambos os gênero, foi identificado $70 \%$ das moças e $51 \%$ dos rapazes em categoria de risco para a saúde quanto aos níveis de atividade física. França (2008), em estudo com escolares de ambos os gêneros do sul do Estado de Sergipe, com idades entre 15 e 18 anos, verificou a ocorrência do sedentarismo em 90, $1 \%$ das moças e $87,0 \%$ dos rapazes. 
Os dados encontrados no levantamento realizado no presente estudo, quando comparados com aqueles obtidos por Farias Júnior (2002) em estudo com escolares de Florianópolis, Santa Catarina, apontam consumo menor de alimentos menos saudáveis no município sergipano. Neste caso, os escolares aqui investigados apresentaram proporção de 21,8\% para o consumo de refrigerantes, 16,4\% de frituras e 34,8\% para o consumo de doces, em comparação com 33,7\%, 19,8\% e 30,8\%, respectivamente, dos escolares de Florianópolis, Santa Catarina. Por outro lado, quando comparado com o estudo de Silva Junior (2005), realizado no município de Aracaju, Sergipe, observou-se resultados similares, com proporções de 23,8\% para o consumo de refrigerantes, 31,9\% para o consumo de doces e 13,3\% para o consumo de frituras. A análise comparativa entre ambos os gêneros aponta maior consumo de alimentos saudáveis nas moças, especialmente frutas e verduras, sendo que o consumo de frutas é mais que o dobro daquele observado nos rapazes.

A ingestão de alimentos considerados não-saudáveis foi bastante elevada e a proporção de escolares que relataram consumo de alimentos ricos em açúcares e gorduras, em ambos os gêneros, com frequência $\leq 3$ vezes/semana, apresentou variação entre 46,4 e 63,3\%, o que caracteriza comportamento pouco saudável, mas aceitável. $\bigcirc$ baixo consumo de alimentos saudáveis (leite e queijo, frutas e verduras) e o elevado consumo de alimentos hipercalóricos têm sido associados a maior predisposição para problemas de saúde. Além disso, os hábitos alimentares adquiridos durante a adolescência têm importantes repercussões no estado de saúde dos indivíduos, tanto em curto quanto em longo prazo (BRANEN; FLETCHER, 1999).

Outro comportamento considerado negativo é o uso de drogas lícitas, que apresentou quantidade preocupante de respostas positivas, tanto no caso de já ter experimentado cigarro quanto bebidas alcoólicas. Os dados mostram que mais de $30 \%$ das moças e dos rapazes já haviam fumado cigarros em algum momento da vida, e mais de $80 \%$ já experimentaram bebidas alcoólicas. Esses resultados são semelhantes aos achados de Matos et al. (1999) em estudo sobre a saúde e os estilos de vida realizado em escolares portugueses entre I I e I 5 anos, em que foi verificado que 31 ,3\% dos jovens do gênero masculino e 31, I \% do gênero feminino referiram já ter experimentado cigarro. No entanto, quando considerado o hábito de já ter experimentado bebidas alcoólicas verificou-se que os valores do presente estudo são superiores aos de Matos et al. ( 1 999), tendo sido identificado prevalência de $77 \%$ para o gênero masculino e $74 \%$ para o gênero feminino.

O V Levantamento Domiciliar sobre o Uso de Drogas Psicotropicas em I 5 capitais do Brasil (VIGESCOLA, 2004) apontou que na cidade de Aracaju, Sergipe, 19,9\% dos rapazes e 14,7\% das moças já haviam fumado cigarro e, 44,2\% e $47,4 \%$, respectivamente, já haviam experimentado bebidas alcoólicas. Comparado 
a esses, as proporções encontradas entre os escolares de Barra dos Coqueiros foram bastante superiores.

Vale ressaltar que no Brasil o tabaco é responsável por cerca de 100 mil mortes/ ano, sendo 30\% delas por câncer (90\% por câncer de pulmão e 97\% por câncer de laringe) e 25\% por doenças do coração (MINISTÉRIO DA SAÚDE, 1988). As bebidas alcoólicas são responsáveis por mais de $90 \%$ das internações por dependência de drogas, além de aparecer em cerca de $70 \%$ dos casos de mortes violentas e acidentes de trânsito (JACKSON; SAMPAIO, 1994).

Quando os dados sobre experimentação de cigarros e bebidas alcoólicas são analisados com os dados de prevalência do consumo dessas substâncias, observase que, apesar dos rapazes terem experimentado cigarro praticamente na mesma proporção que as moças, nenhum deles se tornou fumante regular; no entanto, cerca de metade deles passou a beber regularmente. As moças, além de beberem tanto quanto os rapazes, se tornaram fumantes na proporção de quase daquelas que experimentaram o cigarro.

O estudo com escolares de ensino médio de escolas públicas e privadas de capitais brasileiras, também constatou baixa prevalência de fumantes, da ordem de 14,6\%, sendo 17,4\% dos rapazes e । 1,9\% das moças. Esses dados diferem daqueles observados em Barra dos Coqueiros, Sergipe, a não ser para as moças, em que a proporção é bastante semelhante, mesmo considerando o intervalo de quatro anos entre ambos os levantamentos (VIGESCOLA, 2004).

Outro comportamento investigado, considerado de risco, é o início precoce da atividade sexual que, juntamente com o não uso de preservativos aumenta acentuadamente a exposição das moças para a gravidez precoce, bem como o risco de doenças sexualmente transmissíveis para ambos os gêneros. Não se dispõe de dados para analisar esta questão no Brasil; porém, observou-se tendência semelhante no relatório apresentado por Camarano (1996) que mostra serem as moças mais precoces no início da atividade sexual.

No que se refere às associações entre os comportamentos de risco para a saúde, corroborando com informações disponibilizadas na literatura, escores significativos em linguagem estatística foram observados entre a experimentação do tabaco e o consumo de bebidas alcoólicas com os demais comportamentos de risco (ANDRADE et al., 2003; LOPES, 1998; PAULILO et al., 2001; SCIVOLETTO et al., 1999). Por outro lado, as associações aqui identificadas referentes ao não uso de preservativo e ao relacionamento sexual precoce deve estar vinculada à ocorrência de relações sexuais casuais, fortuitas; portanto, sem qualquer planejamento de proteção contra a gravidez indesejada e as doenças sexualmente transmissíveis. A associação entre o consumo de bebida alcoólica e o não uso de preservativo pode caracterizar situação 
de vulnerabilidade para a contaminação de DST/AIDS, em razão de elevar a possibilidade de ocorrer entre jovens pouco conhecidos, demonstrando comportamento impulsivo, da busca por experimentação de prazeres imediatos, estimulados, muitas vezes, por atitudes de revolta e de não aceitação da realidade.

\section{CONCLUSÕES}

O presente estudo mostrou resultados semelhantes aos da literatura especializada, com os jovens do gênero feminino em geral apresentando maior prevalência de comportamentos de risco, apesar dos índices bastantes elevados de sobrepeso e de sedentarismo dos rapazes investigados. Ainda, permitiu o estabelecimento de associação entre variáveis, corroborando estudos de outros autores e levando a hipótese sobre o impacto de fatores socioambientais.

Os resultados encontrados vêm reforçar a importância de considerar o contexto em que vivem os adolescentes para compreender como eles constroem sua conduta e o modo de agir em sociedade, diante de questões vitais como saúde, educação, trabalho, lazer e outras, relacionadas à qualidade de vida. No entanto, a qualidade de vida também está diretamente relacionada a fatores como padrão adequado de alimentação e nutrição, de habitação e saneamento; bom relacionamento familiar; segurança quanto a oportunidades de educação ao longo de toda a vida e condições de trabalho futuras; ambiente físico limpo; apoio social para famílias e indivíduos; segurança e garantia dos direitos inerentes à cidadania. Assim, a qualidade de vida está voltada para o ambiente social que, por sua vez, engloba uma gama de outros aspectos como ambiente político, econômico e cultural.

Face a essas conclusões, observa-se a necessidade de futuros estudos de corte e de intervenção, com o intuito de acompanhar e analisar fatores associados aos comportamentos de risco encontrados neste estudo, de forma a permitir melhor compreensão de relações dos comportamentos de risco com o ambiente social em que vivem os jovens do município de Barra dos Coqueiros.

Health Behavior in High School Students from Barra dos Coqueiros, Sergipe, Brazil

ABSTRACT: The study examined the prevalence of health risk behaviors of high school students in the public and private networks in the municipality of Barra dos Coqueiros, Santa Luiza Island, Sergipe, Brazil. The referring information was collected through selfadministered questionnaire. The results showed that $89.2 \%$ referred to be sedentary; $32.6 \%$ already tried cigarettes; $41.9 \%$ consume drunken alcoholic; and $9.1 \%$ had 
experienced some type of illicit drug. These results reinforce the need of new studies of population base, in way to allow better understanding of relationships of the risk behaviors with the social environment of the youth.

KEYWORDS: Lifestyle; Adolescence; Health Education.

\section{Comportamiento de Riesgo para la Salud de Escolares de la Enseñanza Secundaria del Barra dos Coqueiros, Sergipe, Brasil}

RESUMEN: Lo objetivo de este estudio fue analizar la prevalencia de comportamiento de riesgo para la salud en escolares de lo enseñanza secundario del Barra dos Coqueiros, isla de Santa Luzia, que recientemente fue vinculado a Aracaju, capital de Sergipe, Brasil. La información fue recogida a través de cuestionario auto administrado. De los escolares analizó, el 89,2\% declaró ser físicamente inactivos, el 32,6\% había probado el tabaco, 41,9\% bebía alcohol con frecuencia, y el 9, 1\% había utilizado algún tipo de drogas ilícitas. Estos resultados refuerzan la necesidad de nuevos estudios de población, para permitir una mejor comprensión de la relación entre el comportamiento de riesgo y el entorno social de los jóvenes.

PALABRAS CLAVE: Estilo de vida, adolescencia; educación para la salud.

\section{REFERÊNCIAS}

ANDRADE, R. G.; PEREIRA, R.A.; SICHIERI, R. Consumo alimentar de adolescentes com e sem sobrepeso do município do Rio de Janeiro. Cadernos de Saúde Pública. Rio de Janeiro, v. 19 , n. 5, p. $1485-95,2003$.

BRANEN, L.; FLETCHER, J. Comparison of college student's current eating habits and recollection of their food practices. Journal of Nutrition Education. London, v. 31, n. 6, p. 304-3 I0, 1999.

CAMARANO, A. A. Gravidez na adolescência. População Brasileira - Como Vai? Instituto de Pesquisa Econômica Aplicada - IPEA, Brasília, v. I, n. 4, p. 7-10, 1996.

CARMO, M. B.; TORAL, N.; SILVA, M. V.; SLATER, B. Consumo de doces, refrigerantes e bebidas com adição de açúcar entre adolescentes da rede pública de ensino de Piracicaba, São Paulo. Revista Brasileira de Epidemiologia. São Paulo, v. 9, n. I , p. I 2 I - 130, 2006.

CASTRO, I. R. R.; CARDOSO, L. O.; ENGSTROM, E. M.; LEVY, R. B.; MONTEIRO, C. A. Vigilância de fatores de risco para doenças não transmissíveis entre adolescentes: experiência da cidade do Rio de Janeiro, Brasil. Cadernos de Saúde Pública. Rio de Janeiro, v. 24, n. 10 , p. 2279-2288, 2008.

CEBRID. II Levantamento domiciliar sobre o uso de drogas psicotrópicas no Brasil. Estudo envolvendo as 108 maiores cidades do país, 2005. São Paulo: Centro Brasileiro de Informações sobre Drogas Psicotrópicas. Universidade Federal de São Paulo, Escola Paulista de Medicina, Departamento de Psicologia. 2006. 
CROCKER, P. R.; BAILEY, D. A.; FAULKNER, R. A.; KOWALSKI, K. C.; MCGRATH, R. Measuring general levels of physical activity: preliminary evidence for the Physical Activity Questionnaire for Older Children. Medicine and science in sports and exercise, Baltimore, v. 29, p.1344-1349, 1997.

FARIAS JÚNIOR, J. C. Estilo de vida de escolares do ensino médio no município de Florianópolis, Santa Catarina, Brasil. 2002. I 35 f. Dissertação (Mestrado em Educação Física) - Centro de Desportos, Programa de Pós-Graduação em Educação Física, Universidade Federal de Santa Catarina, Florianópolis, 2002.

FRANÇA, M. A. R. Comportamentos associados à ocorrência de baixo peso e sobrepeso em estudantes do sul de Sergipe. 2008. I 44 f. Dissertação (Mestrado em Saúde e Ambiente) - Programa de Pós-Graduação em Saúde e Ambiente, Universidade Tiradentes, Aracaju, 2008.

GALDURÓZ, J. C. F; NOTO, A. R.; FONSECA, A. M.; CARLINI, E. A. V. Levantamento nacional sobre o consumo de drogas psicotrópicas entre estudantes do ensino fundamental e médio da rede pública de ensino nas 27 capitais brasileiras: 2004. São Paulo: Centro Brasileiro de Informações sobre Drogas Psicotrópicas - CEBRID. Departamento de Psicobiologia da Escola Paulista de Medicina. Universidade Federal de São Paulo. 2005.

GUEDES, D. P.; GUEDES, J. E. R. P.; BARBOSA, D. S.; OLIVEIRA, J. A. Níveis de prática de atividade física habitual em adolescentes. Revista Brasileira de Medicina do Esporte. Rio de Janeiro, v. 7, n. 6, p. I87-200, 2001.

GUEDES, D. P.; LOPES, C. C. Validação da versão brasileira do Youth Risk Behavior Survey 2007. Revista de Saúde Pública, São Paulo, v. 44, n. 5, p. 840-850, 2010.

IBGE. Pesquisa sobre dados demográficos do Estado de Sergipe. Instituto Brasileiro de Geografia e Estatística. Rio de Janeiro. 2008.

JACKSON, J.; SAMPAIO, C. Saúde Mental. In: ROUQUAYROL, M. Z. Epidemiologia e Saúde. São Paulo: Manole. 1994.

LOPES, G. P. A contracepção na adolescência os desafios do $3^{\circ}$ milênio, relativos à América Latina. Jornal FEBRASGO, São Paulo, v. 9, n. 10, p. 7, 1998.

MALCON, M. C.; MENEZES, A. M. B.; CHATKIN, M. Prevalência e fatores de risco para tabagismo em adolescentes. Revista de Saúde Pública. São Paulo, v. 37, n. I , p. I-7, 2003.

MATOS, M. G. Saúde e estilo de vida em jovens portugueses em idade escolar. IN SARDINHA, L.B.; MATOS, M. G.; LOUREIRO, I. (Eds.). Promoção da saúde: modelos e práticas de intervenção nos âmbitos da actividade física, nutrição e tabagismo. Lisboa: Edições FMH, 2005. v. I, p.217-240.

MINISTÉRIO DA SAÚDE. Divisão nacional de doenças crônico degenerativas. Projeto saúde: Estudo sobre o estilo de vida. São Paulo: Levantamentos Pesquisas e Marketing. 1988.

OMS - Programación para la salud y el desarrollo de los adolescentes. Organización Mundial de La Salud, Ginebra, 1999. 
PAULILO, M. A. S.; JEOLÁS, L. S.; URAHAMA, C. K.; CAMPANERI, M. A. R.; LIMA, M. L. Risco e vulnerabilidade: jovens e drogas. Semina: Ciência Sociedade Humana. Londrina, v. 22, p. 57-66, 2001 .

PEDEN, M. World Report n Child Injury Prevention. Geneva: World Health Organization; UNICEF. 2008.

PNUD. Atlas do Desenvolvimento Humano no Brasil. Programa das Nações Unidas para o Desenvolvimento /Instituto de Pesquisa Econômica Aplicada/Fundação João Pinheiro. 2003.

ROSA, J. F.; CORREA, M. M. C.; SOUZA, R.; SILVA, R. J. S. Comportamentos de risco e nível de atividade física em crianças e adolescentes de escolas da rede estadual de ensino da cidade de Aracaju (SE). Cadernos de Graduação. Aracajú, v. 5, n. 5, p. 63-76, 2007.

SANTOS, J. R.; SILVA, R. J. S. Prevalência de sedentarismo em jovens escolarizados do município Barra dos Coqueiros. Cadernos de Graduação, Aracajú, v. 6, n. 6, p. 21 -30, 2007.

SCIVOLETTO, S.; TSUJI, R. K.; ABDO, C. H. N.; QUEIRÓZ, S.; ANDRADE, A. G.; GATTAZ, W. F. Relação entre consumo de drogas e comportamento sexual de estudante do segundo grau de São Paulo. Revista Brasileira de Psiquiatria. São Paulo, v. 21 , n. 2, p. 87-94, 1999.

SILVA JÚNIOR, A. G. Comportamentos relacionados à saúde em escolares do ensino médio em Aracaju. Sergipe, Brasil. PPGM/UFS, Aracaju, SE, Brasil, 2005.

SILVA, R. C. R.; MALINA, R. M. Nível de atividade física em adolescentes do Município de Niterói, Rio de Janeiro, Brasil. Cadernos de Saúde Pública. Rio de Janeiro, v. I6, n. 4, p. I09| 1097, 2000.

STRAUCH, E. S.; PINHEIRO, R. T.; SILVA, R. A.; HORTA, B. L. Uso de álcool por adolescentes: estudo de base populacional. Revista de Saúde Pública. São Paulo, v. 43, n. 4, p. 647-655, 2009.

VIGESCOLA. Inquérito domiciliar sobre comportamentos de risco e morbidade referida de doenças e agravos não transmissíveis. Brasília, I 5 capitais e Distrito Federal, 2002-2003. Rio de Janeiro: Ministério da Saúde e Instituto de Câncer, 2004.

Recebido: 28 mar. 2010 Aprovado: 28 set. 2010

Endereço para correspondência: Instituto de Tecnologia e Pesquisa Laboratório de Planejamento e Promoção da Saúde Av. Murilo Dantas 300 - Farolândi Aracajú - Sergipe CEP: 49032-490 